\title{
DiBATTITI FILOSOFICI E SCIENTIFICI SULLA GEOMANZIA NEL MEDIOEVO LATINO
}

\author{
Alessandro Palazzo ${ }^{1}$
}

\begin{abstract}
Riassunto: La geomanzia, una disciplina divinatoria importata dal mondo arabo, prosperò nel medioevo latino. Di fronte a tale popolarità traduttori e autori di trattati geomantici, filosofi, teologi e letterati si interrogarono sulla sua validità, sulle sue implicazioni filosofiche e sulla visione del mondo che essa presupponeva. Il presente contributo esamina questo dibattito. In particolare, si ricostruisce lo statuto epistemologico della geomanzia nei suoi rapporti con l'astrologia. Il problema della scientificità della geomanzia viene discusso anche nel contesto dell'azione della causalità celeste sul mondo sublunare. Sono esaminati i ruoli del geomante e dell'interrogante (quaerens) ed è analizzato il concetto di intentio, che rimanda ad un'analisi psicologica peculiare della geomanzia. Caratterizza la geomanzia anche una dimensione rituale fatta di prescrizioni preliminari e di invocazioni da rivolgere alla divinità. Quanto detto impedisce di ridurre la geomanzia ad una semplice tecnica divinatoria; essa appare piuttosto una forma di sapienza rivelata solo al geomante esperto e moralmente degno, che attinge così la conoscenza di eventi futuri e cose nascoste. Da questa breve ricostruzione emerge l'articolata natura della geomanzia, contraddistinta, nella sua originaria versione araba, da una marcata impronta religiosa e da uno stretto, benché ambiguo, nesso con la profezia Islamica.
\end{abstract}

Parole chiave: Geomanzia. Astrologia. Ugo di Santalla. Gugliemo di Moerbeke. Tommaso d'Aquino.

\section{INTRODUZIONE}

Nel XII secolo la penisola iberica fu la sede di un'ondata di traduzioni di testi filosofici e scientifici greci e arabi. Questo imponente trasferimento di fonti, che ha pochi eguali nel corso della storia, trasmise all'Occidente Latino la geomanzia, una disciplina divinatoria di origine $\operatorname{araba}^{2}$. Le prime traduzioni latine di trattati geomantici arabi risalgono infatti al XII secolo.

${ }^{1}$ Professore Associato, Dipartimento di Lettere e Filosofia, Università degli Studi di Trento, Trento Italia. (D) https://orcid.org/0000-0003-1412-0962 E-mail: alessandro.palazzo@unitn.it

2 P. TAnnery, «L'introduction de la géomancie en Occident et le traducteur Hugo Sanccelliensis», II, in ID., Le Rabolion, in Mémoires Scientifiques, vol. IV, Édouard Privat-Gauthier-Villars, ToulouseParis 1920, 318-53: 319-20. Secondo un'ipotesi, che non sembra però supportata dalle necessarie

http://dx.doi.org/10.1590/0101-3173.2019.v42esp.03.p31

This is an open-access article distributed under the terms of the Creative Commons Attribution License. 
Nella tradizione araba il metodo geomantico fu designato in vario modo, ora come «Darb al-raml» (colpire o gettare la sabbia), come «Khațt al-raml» (la linea della sabbia o scrivere la sabbia), e soprattutto come "Ilm al-raml», che significava "scienza della sabbia»" ${ }^{3}$ e che pare essere stata la denominazione più comune.

I traduttori latini si trovarono di fronte al problema di rendere in latino questa espressione araba. Ugo di Santalla, al quale è tradizionalmente attribuita la prima versione in latino di un trattato arabo di geomanzia - l'Ars geomantiae -, ricorse al termine "geomantia»"

Era, questo, un sostantivo latino di origine greca già in uso da secoli per designare una delle arti divinatorie basate sui quattro elementi. Questa forma di divinazione, attestata in fonti medievali (Isidoro di Siviglia e Rabano Mauro), risaliva all'Antichità (è menzionata anche da Varrone), e non aveva alcun rapporto con il nuovo procedimento descritto nei trattati arabi ${ }^{5}$. Alcune testimonianze precisavano che la geomanzia della tradizione antica era una pratica divinatoria basata sull'osservazione e volta all'interpretazione dei segni della terra, specialmente dei terremoti ${ }^{6}$.

La soluzione lessicale adottata da Ugo ebbe successo sia perché il termine "geomantia» sarebbe diventato di uso comune per designare la nuova arte divinatoria araba - «ilm al-raml» - sia perché le denominazioni alternative («scientia linee», «ars arenaria», «ars punctorum», ecc.) coniate dagli altri traduttori e autori latini di opere geomantiche non si sarebbero affermati.

evidenze storiche, il nome del primo geomante storicamente attestato, Abū 'Abd Allāh Muḥammad ibn 'Uthmān al-Zanātī, tradirebbe un'origine berbera, perché rimanderebbe alla tribù berbera nordafricana di Zanāta: E. Savage-Smith, M. B. Smith, Islamic Geomancy and a Thirteenth-Century Divinatory Device, Undena Publications, Malibu, 1980, 3-4.

${ }^{3}$ T. FAHD, «khațt», Encyclopédie de l'Islam. Nouvelle édition, éd. par E. van Donzel, B. Lewis, Ch. Pellat, Brill, Leiden, 1978, t. IV, coll. 1160-62: 1160; E. Savage-Smith, M. B. Sмith, Islamic geomancy, 1, n. 2; B. Villuendas Sabaté, "Arabic geomancy in Jewish hands. Specimens from the Cairo Genizah", in A. Palazzo, I. Zavattero (ed.), Geomancy and Other Forms of Divination, Sismel - Il Galluzzo, Firenze, 2017 (Micrologus Library, 87), 271-288: 274. Cf. anche T. FAHD, La divination arabe. Etudes religieuses, sociologiques et folkloriques sur le milieu natif de l'Islam, Brill, Leiden, 1966, 196-204: 196-198.

${ }^{4}$ TANnery, «L'introduction de la géomancie en Occident», 318-329.

5 T. Charmasson, Recherches sur une technique divinatoire: la géomancie dans l'Occident médiéval, Librarie Droz-Librarie Champion, Paris-Genève, 1980, 10-12.

' A. Delatte, L. Delatte, «Un traité byzantin de géomancie. Codex Parisinus 2410», in Mélanges Franz Cumont, Université Libre de Bruxelles, Bruxelles, 1936, 575-658: 575. 
Il termine "geomantia» nel suo nuovo significato fu recepito nelle divisioni della filosofia già nel XII secolo e fu poi assunto dalle guide degli studenti circolanti nel XIII secolo ${ }^{7}$.

Nel De divisione philosophiae, Gundisalvi (ca. 1115 - post 1190), seguendo il testo di Isidoro $^{8}$, incluse la geomanzia tra le forme di predizione basate sull'esame degli elementi. A dispetto della collocazione e della denominazione tradizionali, è chiaro che il riferimento era alla tecnica divinatoria descritta nei trattati arabi tradotti in latino, perché la geomanzia veniva associata anche ad altre «sciencie iudicandi» di importazione, come la chiromanzia e la spatulomanzia 9 .

Nel mondo latino la geomanzia ebbe un posto di primo piano tra le discipline divinatorie. L'elevato numero dei manoscritti geomantici - che si aggirano intorno ai 150 secondo i calcoli di Thérèse Charmasson - è il primo indice della rilevanza di questa arte divinatoria. La centralità della geomanzia è evidenziata anche da altri fattori: la presenza di testi geomantici nei cataloghi delle biblioteche medievali; la menzione delle opere geomantiche nella letteratura antidivinatoria; le testimonianze letterarie dell'uso della geomanzia; il rilievo dei possessori dei manoscritti geomantici; la circolazione

7 Cf. Arnvlfus Provincialis, Divisio scientiarum, ed. C. Lafleur, in Quatre introductions à la philosophie au XIII siècle. Textes critiques et études historiques, Institut d'Études Médiévales, Montreal Vrin, Paris, 1988, 319,253-55: "Geomantia et diuinatio facta circa negotia terrena, ut cum inquiritur de itinere faciendo uel de furto inueniendo", che sono due dei temi solitamente affrontati negli elenchi di questioni contenuti nei trattati geomantici. Cf. F. FürBETH, "Die Stellung der artes magicae in den hochmittelalterlichen 'Divisiones philosophiae'», in U. Schaefer (hrsg. v.), Artes im Mittelalter, Akademie Verlag, Berlin, 1999, 249-262.

${ }^{8}$ Isidorus Hispalensis, Etymologiarum sive originum libri XX, ed. W. M. Lindsay, Clarendon, Oxford, 1911, VIII, 9, 13,6-8.

9 Dominicus Gundissalinus, De divisione philosophiae, ed. L. Baur, Aschendorff, Münster i. W., 1903 (Beiträge zur Geschichte der Philosophie des Mittelalters, 4,2-3), 119,21-120,4 (oggi è disponibile una nuova edizione dell'opera con traduzione tedesca a cura di A. Fidora, D. Werner, De divisione philosophiae. Über die Einteilung der Philosophie, Lateinish-Deutsch, Herder, Freiburg i.B. 2007). Sulle arti divinatorie in quest'opera, cf. A. FIDORA, «Der wissenschaftliche Ort der Mantik in der Schule von Toledo (12. Jahrhundert)", in Mantik, Schicksal und Freiheit im Mittelalter, L. Sturlese (hrsg. v.), Böhlau, Köln-Weimar-Wien 2011 (Beihefte zum Archiv für Kulturgeschichte, 70), 3349: 39-44; A. Fidora, «Mantische Disziplinen als aristotelische Wissenschaft. Der epistemologische Integrationsversuch des Dominicus Gundissalinus», in Id. (hrsg. v.), Die mantischen Künste und die Epistemologie prognostischer Wissenschaften im Mittelalter, Böhlau, Köln-Weimar-Wien, 2013 (Beihefte zum Archiv für Kulturgeschichte, 74), 61-72. 
dei manoscritti ${ }^{10}$; la diffusione di trattati geomantici in volgare ${ }^{11}$. Non si deve trascurare il fatto che autori fondamentali della filosofia e teologia tardo medievali, come Alberto il Grande ${ }^{12}$, Tommaso d'Aquino ${ }^{13}$, Bonaventura da Bagnoregio $^{14}$ e Sigeri di Brabante ${ }^{15}$ presero posizione nei confronti dell'arte geomantica, adottando approcci differenziati e giungendo a conclusioni diverse e talora contrastanti, che andavano dal riconoscimento della dignità di "scienza" alla geomanzia alla sua equiparazione a un'attività oziosa rea di perdersi in questioni di poco conto («frivola»). Inoltre un famoso passo del Purgatorio dantesco (Purg. XIX, 4-6), evocando la pratica geomantica (Quando i geomanti lor Maggior Fortuna / veggiono in orïente, innanzi a l'alba, / surger per via che poco le sta bruna), indusse i commentatori della Commedia a confrontarsi con la natura e la realtà della geomanzia. Infine,

${ }^{10}$ T. Charmasson, «La géomancie médiévale: les traités et leur diffusion», in A. Palazzo, I. Zavattero (ed.), Geomancy and Other Forms of Divination, 5-29: 27-28; EAD., Recherches sur une technique divinatoire, 227-266. Alla luce dei progressi della ricerca le stime di Charmasson relative al numero dei manoscritti vanno riviste perché nuovi testimoni manoscritti sono stati identificati.

${ }^{11}$ Si pensi a P. Meyer, «Traités en vers provençaux sur l'astrologie et la géomancie», Romania, 102 (1897), 225-275; G. Contini, Un poemetto provenzale di argomento geomantico, Librairie de l'Université, Fribourg, 1940; L. Means, "A translation of Martin of Spain's De geomancia», in Lister M. Matheson (ed.), Popular and Practical Science of Medieval England, Colleagues Press, East Lansing, Mich., 1994 (Medieval Texts and Studies, 11), 61-121; S. Bertelli, "Un manoscritto di geomanzia in volgare della fine del secolo XIII", Studi di filologia italiana. Bollettino annuale dell'Accademia della Crusca, 57 (1999), 5-32; K.S. Bernard, Compter, dire et figurer: édition et commentaire de textes divinatoires et maqiques en occitan médiéval, Université Michel de Montaigne, Bordeaux, 2008; E. Rubino, "An Italian Translation of William of Moerbeke's Geomancy", Medioevo. Rivista di storia della filosofia medievale, 42 (2017), 8198. Per un primo accesso alla geomanzia in volgare tedesco, cf. F. FürBETH, «Sandrichter und Dämonen in der Geomantie des Mittelalters», in J. Bockmann, J. Gold (hrsg. v.), Turpiloquium. Kommunikation mit Teufeln und Dämonen in Mittelalter und Früher Neuzeit, Königshausen \& Neumann, Würzburg, 2017 (Würzburger Beiträge zur Deutschen Philologie, 41), 161-185: 179-185 e M. Heiles, Das Losbuch. Manuskriptologie einer Textsorte des 14. bis 16. Jahrhunderts, Böhlau, Wien-Köln-Weimar, 2018 (Beihefte zum Archiv für Kulturgeschichte, 83), 105-149.

${ }^{12}$ Cf. Albertus Magnus, Super Porphyrium De V universalibus, ed. M.S. Noya, Aschendorff, Münster i.W., 2004 (Alberti Magni Opera Omnia. Editio Coloniensis, 1.1a), 1, 7, 15,67-70; De mineralibus, II 3 3, ed. A. Borgnet, Paris, 1890 (Opera omnia, 5), 52a; Super Ethica commentum et quaestiones, ed. W. Kübel, Aschendorff, Münster i.W., 1987 (Alberti Magni Opera Omnia, 14,2), 579,24-34.

${ }^{13}$ Cf. Thomas de Aquino, Liber de sortibus ad dominum Iacobum de Tonengo, cura et studio Fratrum Praedicatorum, Roma, 1976 (Sancti Thomae de Aquino Opera Omnia, 43), 203-241, alle pp. 229-38 la versione lunga, alle pp. 239-41 la breve; ID., Summa theologiae, IIa IIae, q. 95, art. 3, sol., Edizioni San Paolo, Cinisello Basalmo (MI), 1988, 1484-85.

${ }^{14}$ Bonaventura, Collationes in Hexäemeron, studio et cura PP. Collegii a S. Bonaventura, ad Claras Aquas, 1891 (Doctoris Seraphici S. Bonaventurae Opera Omnia, 5) IV, 15, 351.

15 Sigerus de Brabantia, Quaestiones in Metaphysicam (reportatio di Cambridge), ed. A. Maurer, Éditions de l'Institut supérieur de philosophie, Louvain-la-Neuve, 1983 (Philosophes Médiévaux, 25), V, 41, 278-87: 282,23-24. 
molteplici e ripetute condanne da parte delle autorità ecclesiastiche, tra le quali va annoverato innanzi tutto il famoso Sillabo del 1277 dell'arcivescovo di Parigi Étienne Tempier, colpirono la geomanzia, confermandone la diffusione e la percepita pericolosità ${ }^{16}$. Questi sono solo alcuni esempi - altri se ne potrebbero fare - che dimostrano quanto la geomanzia fosse al tempo stesso popolare e controversa nel medioevo latino.

Malgrado la sua centralità nella cultura medievale la geomanzia non ha riscosso molto interesse tra gli studiosi contemporanei. Un diffuso pregiudizio, talora inconfessato, ma molto radicato, secondo il quale fu solo una pratica, essendo del tutto priva di qualsivoglia componente teorica, spiega perché scarseggino indagini di carattere storico-filosofico ${ }^{17}$. La storiografia sulla geomanzia medievale latina è molto limitata, soprattutto se confrontata con la ricca produzione sull'astrologia, e composta da rassegne di carattere generale ${ }^{18}$ non interessate alla rilevanza filosofica della questione. A ciò si devono aggiungere studi ed edizioni di singoli testi geomantici in volgare ${ }^{19}$.

${ }^{16}$ La condamnation parisienne de 1277 . Texte latin, traduction, introduction et commentaire par D. Piché, Vrin, Paris, 1999, 76. Sul rapporto tra l'Estimaverunt Indi e la condanna del 1277 mi permetto di rinviare al mio: A. PAlazzo, «L'Estimaverunt Indi et la condanna del 1277», in A. Palazzo, I. Zavattero (ed.), Geomancy and Other Forms of Divination, 167-221, in cui prendo in esame anche altri provvedimenti censori diretti contro le pratiche divinatorie e la geomazia in particolare da parte delle autorità ecclesiastiche. Sulle condanne dell'arte geomantica in ambito occidentale vedi anche quanto scrive FüRBETH, «Sandrichter und Dämonen», 176-179.

${ }^{17}$ Pochi sono gli studi sulla geomanzia medievale latina che si segnalano per un taglio storico-filosofico: dopo le penetranti osservazioni contenute nel pionieristico studio di E. Garin, Lo zodiaco della vita. La polemica sull'astrologia dal Trecento al Cinquecento, Laterza, Bari, 1976, spec. 39-41, e oltre a vari contributi in A. Palazzo, I. Zavattero (ed.), Geomancy and Other Forms of Divination, rimando ad A. Beccarisi, «Natürliche Prognostik und Manipulation: Wilhelms von Moerbekes 'De arte et scientia geomantiae'» in L. Sturlese (hrsg. v.), Mantik, Schicksal und Freiheit im Mittelalter, Böhlau, Köln-Weimar-Wien, 2011 (Beihefte zum Archiv für Kulturgeschichte, 70), 109-127; A. Palazzo, "Geomancy as a Paradigm of Operative Rationality in the Middle Ages", Archiv für Mittelalterliche Philosophie und Kultur, 22 (2015), 55-74.

${ }^{18}$ Segnalo, oltre al già citato volume di CHARMASSON, Recherches sur une technique divinatoire, gli studi di A. K. Ziegler, "Histoire de la géomancie latine du milieu du XII ${ }^{\mathrm{e}}$ siècle au milieu du XVI ${ }^{\mathrm{e}}$ siècle», in Positions des thèses soutenues par les élèves de la promotion de 1934 pour obtenir la diplôme d'archiviste paléographe, École Nationale des Chartes, Paris, 1934, 159-170 e di S. Skinner, Terrestrial Astrology. Divination by Geomancy, Routledge \& Keagan Paul, London-Boston, 1980, 88-119.

${ }^{19}$ Cf. supra n. 11. 
In questa sede intendo invece analizzare lo statuto epistemologico, la validità e i limiti del metodo geomantico nonché i presupposti cosmologici e le implicazioni morali e antropologiche di questa forma di divinazione.

In particolare, sulla base dei primi trattati geomantici tradotti dall'arabo (XII sec.), dei trattati latini posteriori (XIII sec.) e di alcune fonti letterarie e filosofiche (sec. XIII-XIV), il presente contributo è finalizzato a ricostruire il dibattito intorno allo statuto epistemologico della geomanzia nei suoi rapporti con l'astrologia. Il problema della scientificità della geomanzia verrà discusso anche in relazione alla inscrivibilità della tecnica geomantica nel quadro delle influenze celesti sul mondo sublunare, cioè nel contesto della legalità dei processi naturali. Saranno esaminati i ruoli del geomante e dell'interrogante (quaerens) ai fini della riuscita della divinazione geomantica. Un elemento centrale in questo ambito è l'intentio, una nozione complessa che non ha valore morale, ma si articola a più livelli, rimandando ad un processo di analisi psicologica che è peculiare della geomanzia.

\section{GEOMANZIA E ASTROLOGIA: DUE FORME DI PREDIZIONE A CONFRONTO}

Che siano trattati dall'arabo, composti in latino o realizzati in volgare, gli scritti geomantici, generalmente nei prologhi, sollevano la questione della validità del metodo geomantico, soprattutto in rapporto all'astrologia ${ }^{20}$. Era opinione generalmente condivisa che la geomanzia discendesse dall'astrologia. Dato che l'astrologia, si pensava, non era alla portata di tutti a causa della complessità delle sue osservazioni e dei suoi calcoli e della raffinatezza dei suoi strumenti, la geomanzia era in alcuni testi considerata una forma semplificata di astrologia ${ }^{21}$.

Bartolomeo da Parma parla della geomanzia come di «una seconda astrologia abbreviata» (altera astrologia brevis) nata "causa commoditatis in

\footnotetext{
${ }^{20} \mathrm{Si}$ tratta di un dibattito ampio e vivace e documentato non solo dai trattati geomantici, ma anche da fonti teologiche, filosofiche, letterarie: cf. supra note 12-15.

${ }^{21}$ Questa idea è stata recepita con tono dispregiativo anche da certa letteratura contemporanea. La geomanzia araba - ma il giudizio può essere esteso anche a quella latina medievale - è definita da W. van Binsbergen, "The astrological origin of Islamic geomancy", Paper read at: The SSIPS (Society for the Study of Islamic Philosophy and Science) - SAGP (Society of Ancient Greek Philosophy) 1996, 15th Annual Conference: Global and Multicultural Dimensions of Ancient and Medieval Philosophy and Social Thought, 1997 <http://studylib.net/doc/1885 3949/the-astrological-origin-of-islamicgeomancy>, August 2019 (unpublished), 13, «mock astrology of geomancy» e «poor man's astrology».
} 


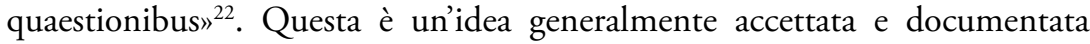
anche in fonti non tecniche: nel Commento al Purgatorio, Jacopo della Lana definisce la geomanzia «astrologia menore» ${ }^{23}$.

Tuttavia, questo processo di semplificazione può essere letto positivamente, e fu infatti letto così. Lungi dall'essere una corruzione dell'astrologia, si sostenne che la geomanzia apportava correzioni ai difetti del metodo astrologico. Il testo che segue l'Ars geomantiae di Ugo di Santalla come suo epilogo nel manoscritto Paris, Bibliothèque nationale de France, lat. 7354, insiste sull'oscurità delle sentenze astrologiche e la loro dispersione in numerosi libri. Tali difetti potrebbero essere superati a condizione di raccogliere le numerose e oscure sentenze in un unico libro con poche utili parole e di aprire la loro oscurità ai confini certi della verità rendendole evidenti. La geomanzia viene così presentata come un'alternativa chiara e sintentica all'astrologia, tanto che lo scopo dell'autore è di svelare i significati delle stelle nelle realtà mondane, in modo rapido e utile, e così di rimuovere dal lettore ogni dubbio in queste cose.

Intentio autem huius auctoris est obscuras et multiplices sententias astronomie utilibus et paucis sermonibus in unum colligere et eorum obscuritatem et tenebrositatem certis limitibus ueritatis aperire et ad lucem id est ad maiorem euidentiam reuocare. Causa intentionis est difficultas astronomie et eius librorum multiplicitas. Multi enim conati sunt iudicia astrorum et significationes tam in bono quam in malo certis rationibus et probabilibus argumentis aperire quod in paucis uoluminibus facere* non potuerunt. Iterumque iste ad hoc nititur ut stellarum significationes in rebus mundanis breniter et utiliter nobis aperiat et omnem scrupulum dubitationis in his a nobis longe repellat $2^{24}$.

I limiti propri dell'astrologia sono in realtà, si precisa ulteriormente nell'epilogo, la conseguenza dei difetti connaturali alla mente umana: gli esseri umani sono incapaci di apprendere la scienza degli astri nella sua interezza a

22 Bartolomaeus Parmensis, Breviloquium artis geomantiae, citato in Charmasson, Recherches sur une technique divinatoire, 14: "Causa, quae ars ista fuit inventa, multiplex fuit, videlicet causa necessitatis, propter consilium habendum, et causa commoditatis in quaestionibus, quae moventur ab hominibus secundum sententiam et doctrinam scientiae illius artis, quae nominatur geomantia, quae est altera astrologia brevis [...]».

${ }^{23}$ Cf. infra, n. 47.

${ }^{24}$ Epilogus Hugonis Sanctellensis Artis geomantiae, in P. Arfé, «L'Ars geomantiae di Ugo di Santalla: il testo e la sua tradizione», A. Palazzo, I. Zavattero (ed.), pp. 65-91: 89,4-14. 
causa della debolezza dei sensi e dell'intelletto ${ }^{25}$. La geomanzia consente all'uomo di accedere alla scienza delle stelle perché stabilisce una corrispondenza tra le 28 case lunari (mansiones) e le sedici figure geomantiche, e a ciascuna delle figure vengono attributi significati relativi alla sfera umana. Considerata da questa punto di vista, l'operazione di sintesi e chiarificazione operata dalla geomanzia risulta funzionale ad un processo di adeguamento del sapere astrologico alla capacità di apprendimento umano. Più immediatamente della scienza delle stelle, la geomanzia fornisce indicazioni di condotta nella vita quotidiana, indicazioni che si ricavano dagli elenchi delle risposte alle questioni geomantiche. Questa è una delle ragioni del grande successo di cui questa pratica divinatoria godette nel medioevo.

Nel prologo al Breviloquium, Bartolomeo da Parma sottolinea che la geomanzia, figlia dell'astrologia (fliam artis astrologie), è libera dai tanti libri e strumenti che invece contraddistinguono la scienza delle stelle:

Volens artem geomantie pratticam quidam seu filiam artis astrologie connnaturate $[s i c]$ inter septem artes liberales bene addiscere prescire debet quod ipsam geomantiam per omnia et in omnibus sequitur manifestissime a stellis licet per alium modum et libera sine tot libris ac instrumentis ${ }^{26}$.

A differenza dell'astrologia, la geomanzia non richiede strumenti complessi, calcoli astrusi o attente osservazioni astrali ${ }^{27}$. Al riguardo è paradigmatica l'affermazione, contenuta nel prologo della Geomantia attribuita a Guglielmo di Moerbeke, secondo la quale il metodo geomantico è creato perché l'ars dell'astrologia tramandata è eccessivamente difficile e prolissa nel formulare i suoi giudizi.

Et ratio huius est ab auctoribus et philosophis huius scientie inventoribus assignata, quoniam ars astronomie nimis erat difficilis et prolixa ad iudicandum tradita. Et iterum quia non in omnibus locis neque in omnibus temporibus licebat illam formare tum propter temporis brevitatem, tum

\footnotetext{
${ }^{25}$ Epilogus Hugonis Sanctellensis Artis geomantiae, ed. P. Arfé, p. 90,39-41: «[...] homines non posse peruenire integraliter et perfecte ad scientiam astrorum tum propter paucitatem sensus tum propter debilitatem ingenii [...]».

${ }^{26}$ Bartolomaeus Parmensis, Breviloquium artis geomantiae, München, Bayerische Staatsbibliothek, $\mathrm{Clm} 398$, f. $1 \mathrm{r}$.

${ }^{27}$ Charmasson, Recherches sur une technique divinatoire, 13-14.
} 
propter incognitum seu inattingibilem stellarum cursum aut planetarum motum sive pro directione vel retrogradatione casum seu stationem ${ }^{28}$.

Inoltre all'astrologo non è sempre e ovunque possibile formulare predizioni sia perché il tempo a sua disposizione per le osservazioni è breve sia perché il corso delle stelle e il moto e lo stato dei planeti sono ignoti e inconoscibili. La procedura astrologica è appesantita da condizioni di carattere empirico e insuperabili limiti oggettivi che minano l'accuratezza della previsione.

Nel prologo all'Ars geomantiae Ugo di Santalla asserisce che una più erudita setta di sapienti, che si dedicarono all'arte del calcolo e volsero l'occhio della mente a perscrutare i segreti dell'astronomia - i luoghi degli astri, le loro orbite dirette, le retrogradazioni, il sorgere, il tramontare, le sublimationes, le depressiones, le alterazioni in questi fenomeni e i prodigi che possono essere ammirati -, individuò molti errori degli astrologi meno sapienti. La ragione costrinse pertanto (hac igitur ratione cogente) gli antichi sapienti (prudens antiquitas) a realizzare (adinuenit) questo compendio - the Ars geomantiae - di tutte quelle conoscenze.

Eruditior prudentium secta, ad computandi artem et astronomie secreta rimanda mentis oculum reuocans, astrorum loca, cursus directos, retrogradationes, ortus, occasus, sublimationes, depressiones et que sunt in his alterationes atque admiranda prodigia attendens, astrologorum minus prudentium multiplicem cognouit errorem. Hac igitur ratione cogente, compendium hoc certissime ex his omnibus prudens adinuenit antiquitas $^{29}$.

${ }^{28}$ Geomantia, in E. Rubino, «Per una edizione della Geomantia di Guglielmo di Moerbeke: il testo del proemio e della prima distinzione della prima parte», in A. Palazzo, I. Zavattero (ed.), pp. 93-134: 102. L'autenticità di quest'opera, già oggetto di controversia, è stata recentemente messa in dubbio da P. Beullens, "Why William of Moerbeke is not the Author of 'His' Geomantia (and Why that does not Make the Text Less Interesting)", Mediterranea. International journal for the transfer of knowledge, 4 (2019), 239-244.

${ }^{29}$ Hugo Sanctellensis, Prologus super Artem geomantiae, in P. Arfé, «Le dimore delle stelle nel Prologus super Artem geomantiae di Ugo di Santalla», in A. Straface, C. De Angelo, A. Manzo (a cura di), "Labor limae. Atti in onore di Carmela Baffioni", Studi Magrebini, n.s. 12-13 (2014-15), I, 41-71: 65,15-21. Sull'Ars geomantiae e le altre traduzioni di Ugo di Santalla, TANnery, "L'introduction de la géomancie», 324-353; C. H. Haskins, Studies in the History of Mediaeval Science, Harvard University Press, Cambridge, 1924, 67-81; J. M. GázQuez, The Attitude of the Medieval Latin Translators Towards the Arabic Sciences, Sismel - Edizioni il Galluzzo, Firenze, 2016 (Micrologus' Library, 75), 51-61. 
Una lettura non superficiale di tutte queste testimonianze corregge il luogo comune che vuole la geomanzia come una pratica divinatoria nata da un processo di mera semplificazione dell'astrologia. In realtà, il rapporto tra queste forme di previsione del futuro è più complesso, perché i testi esaminati evidenziano i limiti della scienza degli astri, che la geomanzia è chiamata a correggere. Per rettificare l'equivoco, oggi largamente diffuso, secondo il quale la geomanzia fu solo una pratica divinatoria priva di qualsivoglia componente teorica, può essere utile applicare anche alla geomanzia latina la distinzione proposta da Toufic Fahd a proposito della tradizione islamica tra la pratica popolare rudimentale della geomanzia e l'arte divinatoria geomantica di grande complessità ${ }^{30}$.

\section{LA SCIENZA GEOMANTICA}

Spesso sommariamente descritta come l'arte consistente nel tracciare immagini e trarne significati relativamente ad eventi futuri e a cose nascoste, la geomanzia è una forma di divinazione non riducibile all'aspetto meramente operativo, perché è articolata in più fasi, è sorretta da una robusta inteleiatura concettuale, analoga a quella astrologica, e prevede una componente di ermeneutica psicologica che sembra preludere alla psicologia del profondo.

1. Ma procediamo per gradi. Nella prima fase, quella operativa, il geomante, su sollecitazione dell'interrogante, che chiede consiglio su una questione specifica che lo riguarda, traccia sulla sabbia, sulla carta o su materiale affine (farina, polvere, ecc.) sedici linee di punti senza contarli, procedendo da destra a sinistra al modo degli Arabi. Il geomante elimina a due a due i punti di ciascuna linea, finché non ne rimangono solo uno o due. Dai resti delle linee, divise per quattro, il geomante ricava quattro figure, che sono in realtà degli schemi di punti (singoli o coppie). Le quattro figure così ottenute sono poi collocate nei primi quattro settori in alto a destra all'interno del tableau geomantico, un'area a forma di piramide rovesciata suddivisa in quindici settori (mansiones). Le prime quattro figure sono dette matres perché il geomante ricava da esse, secondo determinate regole, le figure che occuperanno le altre mansiones (filiae, neptes, iudices, iudex) La risposta alla domanda posta al geomante sta nella quindicesima mansio, la figura giudice. ${ }^{30}$ FAHD, «khatț», col. 1160: "Derrière cette pratique populaire rudimentaire, se cache une arte
divinatoire d'une très grande complexité». 
Se nemmeno la figura giudice scioglie i dubbi, il geomante si trova costretto a ricavare una sedicesima figura, il supergiudice ${ }^{31}$.

2. Pur nella sua apparente semplicità e schematicità, il metodo geomantico è in realtà fondato su un articolato sistema algebrico di carattere binario. I costituenti minimi di ciascuna figura geomantica sono il punto singolo e la coppia di punti, cioè "par et impar». Da elementi fondamentali possono essere formati sedici possibili schemi geomantici ${ }^{32}$. Il tema geomantico è derivato in maniera necessaria dalle quattro figure madri attraverso semplici regole. $\mathrm{E}$ interessante notare che la costruzione del tableau geomantico, pur del tutto casuale, sottostà ad alcuni vincoli imposti dal formalismo matematico, secondo i quali è impossibile per alcune figure comparire più di una volta ed è necessario che la figura giudice sia pari. La complessità dell'aspetto formale del metodo geomantico è stata debitamente messa in luce dagli studi di Robert Jaulin ${ }^{33}$, di Wim van Binsbergen ${ }^{34}$, Maino Pedrazzi ${ }^{35}$ e Marion B. Smith ${ }^{36}$. Anche se questi studi vertono, con l'eccezione di quello di Pedrazzi, sulla geomanzia islamica, le analisi in essi contenuti, proprio per il loro contenuto formale, possono essere applicate alla procedura geomantica in uso anche in Occidente.

3. Ciascuna delle sedici figure geomantiche, identificata da un nome, è stimata favorevole o sfavorevole, è considerata maschile o femminile, è associata ad uno dei quattro elementi ed assume connotazioni astrologiche. A ciascuna figura vengono attribuiti una serie di significati registrati in cataloghi

${ }^{31}$ Sulla costruzione delle figure e del tema geomantico, cf. CHarmasson, Recherches sur une technique divinatoire, 21-27; EAD., «La géomancie médiévale: les traités et leur diffusion», 8-26.

32 Charmasson, Recherches sur une technique divinatoire, 28-33, oltre trascrivere le denominazioni delle sedici figure nella tradizione islamica, riporta un elenco comparativo dei nomi delle figure delle principali traduzioni arabo-latine e dei maggiori trattati latini. L'Estimaverunt Indi attribuisce alle sedici figure i seguenti nomi: Tutela exiens, Tutela intrans, Canus ridens, Diminutus, Rubeus, Albus, Coniunctio, Comprehensum intus, Comprehensum foris, Limen exiens, Limen intrans, Mundus facie, Carcer, Imberbis, Populus, Via.

${ }^{33}$ R. Jaulin, "Essai d'analyse formelle d'un procédé géomantique», Bulletin de l'Institut Français d'Afrique Noire (series B), 19 (1957), 43-71; ID., La géomancie: analyse formelle. Notes mathématiques de F. Dejean, R. Ferry, Mouton \& CO, Paris-La Haye, 1966.

${ }^{34} \mathrm{~W}$. van BinsBergen, «The astrological origin of Islamic geomancy», 5-10.

${ }^{35}$ M. Pedrazzi, «Le figure della geomanzia: un gruppo finito abeliano», Physis. Rivista internazionale di storia della scienza, 14 (1972), fasc. 2, 146-161.

${ }^{36}$ Marion B. SMith, «The Nature of Islamic Geomancy with a Critique of a Structuralist's Approach», Studia Islamica, 49 (1979), 5-38, che ha formulato critiche severe allo studio La géomancie: analyse formelle di Jaulin. Contrariamente alla presunta superiore complessità dell'astrologia, Smith concede che il calcolo degli oroscopi presenta problemi, ma rivendica alla geomanzia maggiore varietà dei metodi di interpretazione e una struttura matematica astratta assente nella scienza degli astri (9). 
di interpretazione, che possono variare da trattato a trattato. L'ampiezza dell'area semantica di ciascuna figura rende più complessa l'interpretazione ${ }^{37}$.

La figura va letta in rapporto alla mansio nella quale è collocata. Ciascuna delle quindici mansiones è posta in relazione a uno o più ambiti della realtà ${ }^{38}$. I trattati divergono anche sui significati delle mansiones. Nel formulare il suo responso, il geomante dovrà esaminare la figura contenuta nella mansio pertinente all'ambito toccato dalla richiesta e verificare se quella figura sia favorevole in sé e in rapporto a tutti gli altri fattori che incidono sul suo significato. Il geomante dovrà sempre prendere in esame anche la prima mansio, relativa all'anima dell'interrogante ${ }^{39}$.

Fattori che alterano il significato delle figure (proprietas, locus, aspectus, motus, paternitas) e complesse procedure di interpretazione, come l'individuazione della casa che sintetizza il tema geomantico (pars fortunae) e il calcolo che stabilisce la via del punto (via puncti), complicano il metodo divinatorio ${ }^{40}$. Come si evince da quanto detto, il responso geomantico discende da un complesso procedimento divinatorio e presuppone un divinatore esperto e competente, per cui non è affidato esclusivamente al caso: la geomanzia non va confusa con le sorti ${ }^{41}$.

4. Pur non ricorrendo ad osservazioni astrali e non avendo per questo bisogno di strumenti né di tavole astronomiche, la geomanzia non è immune da commistioni astrologiche, che, con il trascorrere del tempo e il crescere della consapevolezza metodologica, diventeranno parte integrante della pratica e della teoria geomantiche. Tipico esempio di questi prestiti astrologici è il ricorso a nozioni come conjunctio, aspectus, triplicitas, etc. In generale, come il trattato tradotto da Ugo di Santalla insegna, la geomanzia si costruisce

${ }^{37}$ Charmasson, Recherches sur une technique divinatoire, 28-48.

${ }^{38}$ Per un esempio dei significati delle mansiones, si veda la tabella relativa alla Geomantia attribuita a Moerbeke contenuta in BECCARISI, «Natürliche Prognostik und Manipulation», 125-127.

${ }^{39}$ Charmasson, Recherches sur une technique divinatoire, 48-53.

${ }^{40}$ Charmasson, Recherches sur une technique divinatoire, 53-65. I. ZavatTero, «Estimaverunt Indi: la tradizione testuale di un anonimo trattato di geomanzia», in A. Palazzo, I. Zavattero (ed.), Geomancy and Other Forms of Divination, 31-63: 42-51; EAD., "Estimaverunt Indi: la diffusion d'un text geomantique condamné», Medioevo. Rivista di storia della filosofia medievale, 42 (2017), 57-80: 66-75. Il significato di una figura può essere modificato in congiunzione con altre figure. Per ciò che concerne Populus, cf. Estimaverunt Indi, Firenze, Biblioteca Medicea Laurenziana, Plut. XXX.29, ff. 15va-16ra.

${ }^{41}$ Le osservazioni di Josefina Rodríguez-Arribas su questo punto possono essere applicate anche alla geomanzia latina: cf. J. Rodríguez-Arribas, «Divination According to Goralot: Lots and Geomancy in Hebrew Manuscripts», in A. Palazzo, I. Zavattero (ed.), Geomancy and Other Forms of Divination, 243-270: 248-269, 
sulla base di un esatto rispecchiamento del mondo superiore («in superiori circulo», «superioris regionis») nel mondo sublunare («in hoc mundo», «hoc inferius» $)^{42}$. Dalla perfetta simmetria tra il cielo e la terra, Ugo fa derivare le figure geomantiche dai segni zodiacali e dalle mansioni lunari, entro cui le mansioni si trovano. "Sicque manifestum est quod huiusmodi figure, quas hic prosequi uolumus, signorum pariter et lunarium mansionum formas omnino sequuntur" ${ }^{33}$.

Per questa ragione le immagini geomantiche vengono investite del potere dei segni zodiacali e dei pianeti, che sono i signori dei segni.

Figure huiusmodi namque ex mansionibus procedentes, signorum naturam ex quibus procedunt eorumque motus et dominorum qui eisdem presunt discursus, fortunam, necnon et infortunium penitus secuntur ${ }^{44}$.

Questa cura nel creare simmetrie non è prerogativa dell'Ars geomantiae, ma caratterizza anche altri trattati geomantici, che si trovano di fronte al problema della discordanza numerica tra i segni dello zodiaco, che sono dodici, e i pianeti, che sono sette, da un lato, e le figure geomantiche, che sono sedici. Per ovviare all'asimmetria segni-figure, quattro segni dello zodiaco vengono fatti corrispondere a due figure geomantiche, mentre la discordanza numerica tra i sette pianeti e le sedici immagini è superata attribuendo ogni pianeta a due immagini. Inoltre, due immagini, Caput e Cauda, godono della prerogativa di essere associate ciascuna a due pianeti ${ }^{45}$.

\footnotetext{
${ }^{42}$ Hugo Sanctellensis, Prologus super Artem geomantiae, ed. P. Arfé, 65-66,22-25. Sul prologo dell'Ars geomantiae e la dipendenza delle immagini geomantiche dalle mansioni lunari e dai segni dello zodiaco, cf. C. BurnetT, "Hermetic Geomancy, "Ratione certis experimentis usitata"», in A. Palazzo, I. Zavattero (ed.), Geomancy and Other Forms of Divination, 135-141. Sempre a proposito del nesso tra le figure geomantiche e gli asterismi lunari nel quadro dell'azione dei cieli sulle realtà inferiori, Burnett nel medesimo contributo fornisce utili ragguagli anche su un'opera poco nota, il Tractatus geomancie di Burnettus di Viella.

${ }^{43}$ Hugo Sanctellensis, Prologus super Artem geomantiae, ed. P. Arfé, 66,25-26. Sulle mansioni lunari, cf. P. Kunitzsch, "al-Manäzil», in The Encyclopaedia of Islam, New Edition, in C.E. Bosworth, E. van Donzel, B. Lewis, Ch. Pellat, W.P. Heinrichs (ed.). vol. VI, Brill, Leiden, 1991, 374-376; Savage-Smith, Smith, Islamic Geomancy 36-43; C. Burnett, «Lunar Astrology. The Varieties of Texts Using Lunar Mansions, with Emphasis on Jafar Indus», Micrologus, 12 (2004), 43-133 (bibliografia, 43, n. 1).

${ }^{44}$ Hugo Sanctellensis, Prologus super Artem geomantiae, ed. P. Arfé, 66,47-50.

${ }^{45}$ Charmasson, Recherches sur une technique divinatoire, 43-45.
} 


\section{IL GEOMANTE E L'INTERROGANTE: AGENTI CONSAPEVOLI O STRUMENTI INCONSAPEVOLI?}

Il paradigma generale della dipendenza del mondo terrestre dal mondo superiore si specifica nei trattati geomantici in osservazioni specifiche sull'influenza esercitata dalle sfere celesti sulle figure geomantiche. Il fatto che i geomanti tengano in considerazione le condizioni meteorologiche e determinati fattori astrologici rafforza la convinzione che la causalità celeste giochi un ruolo decisivo nella concreta attuazione della tecnica geomantica. Ciò porta a sollevare la questione di quale sia il ruolo giocato dai due agenti coinvolti nel procedimento geomantico, il geomante e il consultante. Se agiscano intenzionalmente o siano piuttosto strumenti inconsapevoli delle sfere celesti è una questione centrale della discussione sulla scientificità della geomanzia.

Nel De sortibus, Tommaso scrive che il geomante nell'atto di tracciare le linee («in protractione punctorum») agisce senza premeditazione razionale, mentre il quaerens interroga istigato da una tensione interiore («quasi interiori sollecitudine instigatus ») e non per decisione razionale: «Unde et in protractione punctorum geomantie actores hoc obseruandum putant ut ille qui protrahit puncta absque premeditatione rationis procedat, et ille qui consultat quasi interiori sollecitudine instigatus interroget, non quasi ex deliberatione rationis ${ }^{46}$.

Nella fase operativa iniziale, quella della domanda dell'interrogante e del tracciare le linee da parte del geomante, il metodo geomantico esclude l'intervento delle facoltà superiori dell'anima, cioè l'intelletto e la volontà. Il geomante e il consultante sono strumenti passivi della causalità celeste. Su questo punto Tommaso riflette le idee che sono esposte in alcuni trattati geomantici.

Il commento al Purgatorio di Dante di Jacopo della Lana conferma queste concezioni. Infatti, Jacopo teorizza esplicitamente l'attitudine passiva del geomante e il suo essere uno strumento della efficace influenza delle

\footnotetext{
46 Thomas de Aquino, De sortibus, c. 4, 234,159-164. Sulla spiegazione della geomanzia proposta da Tommaso in quest'opera, cf. P. Porro, «Divinazione e geomanzia in Tommaso d'Aquino: qualche osservazione sul De sortibus", in A. Palazzo, I. Zavattero (ed.), Geomancy and Other Forms of Divination, 143-166; L. Sturlese, "Thomas von Aquin und die Mantik», in Id. (hrsg. v.), Mantik, Schicksal und Freiheit im Mittelalter, Böhlau, Köln-Weimar-Wien, 2011 (Beihefte zum Archiv für Kulturgeschichte, 70), 97-107: 100-104. Sulla dipendenza del geomante e dell'interrogante dalla causalità celeste, cf. le acute osservazioni di A. BecCARIsI, "Guglielmo di Moerbeke e la divinazione», in A. Palazzo, I. Zavattero (ed.), Geomancy and Other Forms of Divination, 371-395: 380, 384-394.
} 
sfere celesti. Commentando la terzina del Purgatorio nella quale compare il riferimento ai geomanti (Purg. XIX, 4-6), Jacopo della Lana precisa che la tecnica geomantica consiste nel tracciare sedici linee di punti a caso («a ventura»), cioè senza contare i punti («cença numerare li punti»). L'operazione è compiuta al momento dell'aurora («in l'aurora»), quando la digestione è completa, e le membra («menbri del corpo»), non impedite («agravadi») da cause interne, sono più ricettive delle influenze dei movimenti celesti.

Jacopo della Lana osserva che il corpo umano può essere mosso da tre cause: da un atto di volizione attivato da una decisione del libero arbitrio; da un atto di volizione determinato dalle affezioni corporee causate dall'eccesso di vino, cibo, ecc.; o da una inclinazione naturale soggetta al movimento della sfera celeste. La prima causa, cioè la decisione del libero arbitrio, esclude la pratica geomantica che richiede che i punti non siano tracciati volontariamente e non siano contati; esiste incompatibilità anche tra le affezioni corporee interne causate dalla digestione ancora in corso e la pratica geomantica, che può avere luogo all'aurora perché solo allora la digestione è davvero ultimata. Solo la terza causa è compatibile con la geomanzia, e per questa ragione la geomanzia è chiamata «astrologia menore» ${ }^{47}$.

Secondo Jacopo, la correttezza della procedura geomantica è garantita solo sel'atto di tracciare i punti non è determinato da fattori razionali o volontari. Il geomante non agisce intenzionalmente, ma è soggetto ai movimenti delle

${ }^{47}$ Iacomo della Lana, Commento alla "Commedia", a cura di M. Volpi con la collaborazione di A. Terzi, 4 tomi, Salerno Editrice, Roma, 2009, Purgatorio 19,4, II, 1312-14: “Quando i geomanti' [...] Mostrado per uno modo l'ora della soa visione, anche lo vol mostrare per un altro e dixe ch'era quell'ora quando li geomanti, çoè quilli che usano geomancia [...] Contra la qual cosa è da savere ch'ell'è un'arte la quale sí se fa in terra overo in sabione over cum penna et incostro suso carte, et fassi xvj linee de punti a ventura, çoè cença numerare li punti; poi de quelle figure sí se tra' quattro figure le qua’ sono appellade madri. Poi de quelle secundo lo rito de quella arte se fano le altre; poi secundo le figure e i aspetti de quelle dano çudisio delle cose a domandare. Or la casone perché li ditti artisti dixeno essere in l'aurora piú adatto lo tempo a soa arte si <è> che in quell'ora omne digestione de cibo è fatta, sí ch'i menbri del corpo no èno agravadi da alcuna casone intrinseca, ma tutti desposti ad ubedire li movimenti celesti. E perché supono essere quî movementi celesti casuni secundo natural corso de quisti inferiuri mutamenti, conchiudeno talora essere abile a loro arte. Pò essere li membri humani mossi da tre casuni: l'una da voluntà della qual nasse da eletione fatta per libero arbitrio; l'altra da voluntà mossa da alcuna passione, come per troppo cibo, per troppo vino, per troppo resolutione d'omuri o per troppo manitione de spirti vitalli; la terça da inclinatione naturale la qual soçiase al movemento del celo, si cume in phylosophia naturale e in astrologia chiaro se pone. La prima casone exclude l'arte della geomancia quando vole ch'i punti no se façano a numero né per voluntà né piú né meno; la secunda casone simelmente exchiude quando accepta so tempo in l'ora che omne digestione si è facta, della quale, com'è ditto, l'autor in lo presente capitulo fa mentione; la terça casone accepta la ditta arte per so principio appellandosse per alcuni l'astrologia menore. E perçò sí dà ad intender l'autore ch'el dixe che lla preditta visione fo circa l'aurora». Il corsivo è mio. 
sfere celesti, che sono la causa degli eventi nella sfera sublunare. Il geomante è lo strumento passivo del fato. Tracciando le linee, egli è la mano della causalità celeste, che di lui si serve per tracciare sedici linee su un supporto materiale (sabbia, polvere, carta, ecc.) e per trasmettere nelle immagini geomantiche l'intentio.

\section{L'INTENTIO: IL CONTENUTO DELLA DIVINAZIONE GEOMANTICA}

La complessità del metodo geomantico e il rilievo teorico delle questioni in campo sono evidenti nel prologo dell'Estimaverunt Indi, uno dei primi trattati arabi ad essere stati tradotti in latino, di cui non conosciamo l'autore né il traduttore, molto noto per aver goduto del triste privilegio di essere stato condannato dal vescovo Tempier nel 1277, unica opera ad essere esplicitamente citata insieme al De amore di Andrea Cappellano ${ }^{48}$.

Il prologo dell'Estimaverunt Indi introduce il concetto chiave di intentio $^{49}$. È una nozione che si traduce con difficoltà perché l'intentio si dispiega su differenti livelli della realtà.

Estimaverunt Indi quod quando lineantur linee absque numero et prohiciuntur pares et eriguntur, ex eo quod remanent figure quattuor, deinde generantur et concluduntur ad inueniendam intentionem, significant illud quod erit, et facit eas necessarias orbis ad illud quod rectum est, et interpretatur de eo quod in anima est.

Nota quod figure disponuntur secundum dispositionem orbis signorum ad illud quod est rectum, quia circulus erit secundum intentionem querentis et cognitionem cordis eius ad illud quod est rectum, et interpretatur de eo quod est in anima.

Come detto, il presupposto fondamentale del metodo geomantico è la dipendenza della regione terrestre dal mondo superiore. Il geomante traccia le linee senza contare i punti («absque numero»). Da ciò che rimane di queste linee egli ottiene le quattro figure madri, dalle quali, in ultima istanza, egli può ricavare l'intentio, predire il futuro ("quod erit») e percepire ciò che è nell'anima dell'interrogante («de eo quod in anima est»). Le linee tracciate sono rese necessarie dall'influenza degli orbi celesti («et facit eas necessarias orbis»),

\footnotetext{
${ }^{48}$ Sulla condanna dell' Estimaverunt Indi, cf. supra n. 16.

${ }^{49}$ Un'edizione provvisoria del testo è al momento disponibile in I. ZavatTero, «Estimaverunt Indi: la tradizione testuale», 59-62.
} 
per cui anche le figure rispecchiano la disposizione della sfera dei segni dello zodiaco ("figure disponuntur secundum dispositionem orbis signorum»). Ma dato che il circolo celeste corrisponde all'intentio del quaerens e rende note le profondità della sua mente ("circulus erit secundum intentionem querentis et cognitionem cordis eius»), allora le immagini geomantiche, disposte secondo la sfera zodiacale, permettono di percepire ciò che si nasconde nell'anima dell'interrogante («quod est in anima»).

In altre parole, la figura geomantica, la mente dell'interrogante e la sfera celeste contengono, ciascuna secondo la propria capacità e in modo diverso, la stessa intentio. Dopo aver tracciato sedici linee senza contarle, cioè mettendo tra parentesi la propria razionalità e quindi sotto l'influenza della causalità celeste, il geomante può leggere l'intentio dell'immagine.

A questo punto diventa chiaro perché il consultante, quando rivolge una richiesta al geomante, deve essere mosso da una tensione interiore istintiva («interiori sollecitudine»), come scrive Tommaso, piuttosto che da consapevole deliberazione. A ciò il consultante è obbligato dal fatto che l'influenza degli astri si riflette nei recessi segreti e profondi della sua anima, al di sotto della superficie dei pensieri razionali e delle riflessioni consapevoli. Si capisce anche perché il geomante debba essere non solo un divinatore e leggere nelle immagini, ma anche uno psicologo del profondo e scrutare nella psiche dell'interrogante, alla ricerca dell'autentico contenuto della sua richiesta, contenuto che è protetto nella parte più recondita dell'anima dell'interrogante. L'attenzione rivolta a scrutare il cuore del quaerens serviva anche a prevenire richieste menzognere e inganni finalizzati a testare le abilità del geomante. Tentativi di frode sono attestati nelle fonti e confermati dalle esortazioni, contenute nei trattati geomantici, a non mettere alla prova le competenze del geomante con false questioni ${ }^{50}$.

Lungi dall'essere una semplice tecnica nata dalla semplificazione dell'astrologia, la geomanzia è in realtà una complessa disciplina divinatoria, che condivide con la più nota astrologia lo schema teorico fondamentale (la dipendenza della regione inferiore dai cieli) e molti concetti specifici, ma innesta su questa base elementi molto eterogenei: un'articolata costruzione delle immagini geomantiche e del tema geomantico, una raffinata ermeneutica divinatoria e un'originale analisi psicologica.

${ }^{50}$ A proposito della tradizione ebraica, cf. Rodríguez-Arribas, «Divination According to Goralot: Lots and Geomancy in Hebrew Manuscripts», 243-244; Charmasson, Recherches sur une technique divinatoire, 20. 
Et scias quod non formantur figure secundum quod intendit querens, nec secundum questionem eius, et non formantur in eis nisi cum eo cuius portendit potentia esse, siue querat de eo querens, aut non querat, sicut formantur in uisione, et propter illud errat in eis qui credit quod intentio egrediatur in eis secundum omnem habitudinem in eis uoluntarie, cum uere res non est ita. Et non formatur intentio in eis nisi quando est in eis quod est possibile esse, et cuius casus est necessarius. Nam intentio illa est que formatur in eis, et quando homo intendit rem cuius esse non pertransit potentia, non formatur in eis nisi quando erit, sicut praemisimus.

L'Estimaverunt insiste sulla dipendenza delle immagini geomantiche dalla causalità celeste. Essendo disposte secondo la sfera dello zodiaco, le immagini geomantiche sono al di fuori del controllo del quaerens. Non sono formate né secondo il suo intendimento né secondo la sua richiesta («non formantur figure secundum quod intendit querens, nec secundum questionem eius»). Sono formate proprio come le immagini sono prodotte in una visione. Ciò significa che l'intentio non penetra («egrediatur») nelle immagini per decisione volontaria («secundum omnem habitudinem in eis uoluntarie»), ma l'intenzione appare solo in quelle immagini nelle quali è possibile che sia, e delle quali è necessario il formarsi («cuius casus est necessarius»).

In un altro passo l'Estimaverunt Indi descrive le grandi capacità di penetrazione dell'arte geomantica, che svela le cose e le loro verità, tutto ciò che di quelle è piccolo o grande, le loro qualità proprie e i loro attributi comuni, e ciò che consegue da tutti gli accidenti nei cuori degli uomini («totum quod accidit ex omnibus accidentibus stantibus in pectoribus hominum») e che è formato nelle loro menti ("formatum in eorum mentibus») ed è in accordo con le intenzioni nelle loro anime ("continuatum cum transitibus eorum accidentibus, idest intentionibus in animabus ipsorum»). È Dio che svela ciò che è nascosto traendolo all'essere e facendolo apparire nella soluzione.

Quando ergo descendit in domibus, detegit tibi uisibilitates rerum et earum ueritates et quod ex eis est minimum et quod ex eis est grande et proprietatem earum et communicationem ipsarum et totum, quod accidit ex omnibus accidentibus stantibus in pectoribus hominum et formatum in eorum mentibus et continuatum cum transitibus eorum accidentibus, id est intentionibus in animabus ipsorum. Et quod uelatum est et occultum extrahit deus de priuatione ad esse et ex occultatione ad apparitionem solutione sana et uirtute ${ }^{51}$.

${ }^{51}$ Estimaverunt Indi, Firenze, Biblioteca Medicea Laurenziana, Plut. XXX.29, f. 4rab. 


\section{Conclusione}

La geomanzia descritta nelle traduzioni latine di testi arabi, nei trattati scritti in latino, in componimenti in volgare e in fonti di varia natura (filosofica, teologica e letteraria) è una disciplina divinatoria complessa. Essa richiede al geomante lunga esperienza, competenze ermeneutiche e abilità psicologiche. Il confronto con l'astrologia, frequente nelle fonti medievali, spesso ha generato fraintendimenti, inducendo gli studiosi a leggerla come una semplificazione della scienza degli astri.

In realtà quel paragone tradisce la competizione tra due differenti forme di divinazione, tra due modelli di predizione ${ }^{52}$. Mentre lo statuto dell'astrologia come scienza è indiscusso e universalmente riconosciuto, riposando su una consolidata tradizione, l'arte geomantica, malgrado la sua diffusione, rimane nel medioevo latino una forma di divinazione controversa e per questo è alla costante ricerca di un riconoscimento sul piano scientifico e sociale. Il confronto polemico con l'astrologia, la più nobile tra le scienze predittive, va spiegato in questi termini. In questa prospettiva diventa comprensibile perché alcuni trattati geomantici evidenzino i limiti strutturali della scienza degli astri, per correggere i quali sarebbe stata inventata la geomanzia. La necessità di accreditare l'arte geomantica conferendole autorevolezza spiega, almeno in parte, anche perché gli scritti geomantici contengano narrazioni sulle origini mitiche e religiose dell'arte geomantica. Si capiscono anche le tante attribuzioni illustri che danno lustro a traduzioni anonime e trattati apocrifi ${ }^{53}$.

Spesso i trattati geomantici indugiano sulle precondizioni richieste per l'avvio della procedura vera e propria, cioè del getto dei punti. Si osserva che è necessario scegliere condizioni atmosferiche favorevoli e tener conto delle configurazioni astrali (la mansio del geomante e la posizione del pianeta che presiede alla materia oggetto della questione). Inoltre, prima di procedere, il geomante deve invocare Dio o Cristo ${ }^{54}$.

\footnotetext{
${ }^{52}$ La contrapposizione tra due modelli di predizione radicalmente diversi è prospettata esplicitamente da Ibn Khaldûn, Discours sur l'histoire universelle. Al-Muqaddima. Traduction nouvelle, préface et notes par V. Monteil, 3 voll., Sindbad, Paris, 1978, I, 222-225.

${ }^{53}$ Charmasson, Recherches sur une technique divinatoire, 227.

${ }^{54}$ Geomantia, ed. E. Rubino, in EAD., "Per una edizione della Geomantia di Guglielmo di Moerbeke», 118: «De tempore vero dicimus sic, quod tempus debet esse serenum, non nebulosum nec aquosum nec ventulosum, cuius ratio dicetur in quinta particula huius tractatus. De hora dicimus sic: Non in altitudine solis; et quod fiat questio de re, cuius planeta dominatur, sicut a tertiis usque ad vesperas». Charmasson, Recherches sur une technique divinatoire, 20-21.
} 
Talora si tratteggia un breve profilo del geomante. Bartolomeo da Parma sostiene che egli deve essere saggio, avere un carattere calmo e sereno, essere abile ed esperto. Dopo aver penetrato tutte le sottigliezze della geomanzia e averne intuito i segreti, sarà in grado di padroneggiare tutto ciò che pertiene alla sua arte. Infine, egli sarà una persona retta. L'interrogante deve essere onesto, avere fiducia nella competenza del divinatore e rivolgerglisi alla ricerca della verità e non con l'intenzione di ingannarlo ${ }^{55}$.

Lungi dall'essere semplici istruzioni procedurali, le indicazioni relative alle invocazioni preliminari e la descrizione della personalità e delle qualità del geomante e dell'attitudine dell'interrogante rimandano a una dimensione rituale che appare la cifra caratteristica della geomanzia. Sarebbe fuorviante ridurre la geomanzia a una semplice tecnica divinatoria; essa appare piuttosto una forma di conoscenza sapienziale che in tanto dischiude eventi futuri e cose nascoste, in quanto il geomante conduce uno stile di vita moralmente irreprensibile. Le qualità morali e spirituali del geomante e l'attitudine del consultante, le invocazioni di Dio da parte del geomante, le linee tracciate senza pensare - senza contare i punti -, la richiesta del quaerens posta su sollecitazione di un impulso non razionale, l'intentio trasmessa dai cieli nella anima del quaerens e riflessa nell'immagine geomantica: sono tutti elementi caratteristici dietro ai quali è possibile discernere l'originale ispirazione religiosa della geomanzia islamica, il nesso tra geomanzia e profezia tipico della tradizione islamica.

PALAZZO, A. Philosophical and scientific debates on geomancy in the Latin Middle Ages. Trans/Form/Ação, Marília, v. 42, p. 31-56, 2019. Edição Especial.

Abstract: Geomancy, a divinatory discipline imported from the Arab world, flourished in the Latin Middle Ages. In the attempt to explain the popularity of geomancy, translators and authors of geomantic writings, philosophers, theologians, and other scholars addressed such issues as its validity, its philosophical implications, and the worldview it presupposed. This paper explores this debate, delineating the epistemological status of geomancy in relation to astrology. The problem of the scientificity of geomacy will be discussed with regard to the celestial influences on the sublunary world. In addition, the different roles played by both the geomancer and the consultant (quaerens) are examined. Attention will be paid to the concept of intentio, which implies a psychological analysis

${ }^{55}$ Charmasson, Recherches sur une technique divinatoire, 19-20. 
peculiar to geomancy. Geomancy is also characterized by ritual elements such as both preliminary prescriptions and invocations of God, meaning that it is not an ordinary divinatory technique, but a form of wisdom revealed only to experienced and upright geomancers, who can thus obtain knowledge of future events and hidden things. From this analysis emerges the complex nature of geomancy, whose original Arabic version had a prominent religious character and a close, albeit ambiguous, link with Islamic prophecy.

KEYwORDs: Geomancy. Astrology. Ugo of Santalla. William of Moerbeke. Thomas Aquinas.

\section{RIFERIMENTI BIBLIOGRAFICI}

ALBERTUS MAGNUS. De mineralibus. Ed. A. Borgnet. Paris: 1890. (Opera omnia, 5).

ALBERTUS MAGNUS. Super Ethica commentum et quaestiones. Ed. W. Kübel. Münster i.W.: Aschendorff, 1987. (Alberti Magni Opera Omnia. Editio Coloniensis, 14,2).

ALBERTUS MAGNUS. Super Porphyrium De V universalibus. Ed. M.S. Noya. Münster i.W.: Aschendorff, 2004 (Alberti Magni Opera Omnia. Editio Coloniensis, 1.1a).

ARNVLFUS PROVINCIALIS. Divisio scientiarum. In: LAFLEUR, C. Quatre introductions à la philosophie au XIII siècle: textes critiques et études historiques. Montreal; Paris: Institut d'Études Médiévales; Vrin, 1988. pp. 297-347.

BARTOLOMAEUS PARMENSIS, Breviloquium artis geomantiae. München: Bayerische Staatsbibliothek, Clm 398, ff. 1-105.

BECCARISI, A. Guglielmo di Moerbeke e la divinazione. In: PALAZZO, A.; ZAVATTERO, I. (ed.). Geomancy and Other Forms of Divination. Firenze: Sismel - Il Galluzzo, 2017. pp. 371-395.

BECCARISI, A. Natürliche Prognostik und Manipulation: Wilhelms von Moerbekes 'De arte et scientia geomantiae'. In: STURLESE, L. (hrsg. v.). Mantik, Schicksal und Freiheit im Mittelalter. Köln-Weimar-Wien: Böhlau, 2011. pp. 109-127.

BERNARD, K. S. Compter, dire et figurer: édition et commentaire de textes divinatoires et maqiques en occitan médiéval. Bordeaux: Université Michel de Montaigne, 2008.

BERTELLI, S. Un manoscritto di geomanzia in volgare della fine del secolo XIII. Studi di Filologia Italiana. Bollettino Annuale dell'Accademia della Crusca, v. 57, pp. 5-32, 1999.

BEULLENS, P. Why William of Moerbeke is not the Author of 'His' Geomantia (and Why that does not Make the Text Less Interesting). Mediterranea. International Journal for the Transfer of Knowledge, v. 4, pp. 239-244, 2019.

BONAVENTURA. Collationes in Hexäemeron. Studio et cura PP. Collegii a S.

Bonaventura. Florentiae, Ad Claras Aquas, 1891. (Doctoris Seraphici S. Bonaventurae Opera Omnia, 5). 
BURNETT, C. Hermetic Geomancy, "Ratione certis experimentis usitata”. In: PALAZZO, A.; ZAVATTERO, I. (ed.). Geomancy and Other Forms of Divination. Firenze: Sismel - Il Galluzzo, 2017. pp. 135-141.

BURNETT, C. Lunar Astrology. The Varieties of Texts Using Lunar Mansions, with Emphasis on Jafar Indus. Micrologus, v. 12, pp. 43-133, 2004.

CHARMASSON, T. La géomancie médiévale: les traités et leur diffusion. In: PALAZZO, A.; ZAVATTERO, I. (ed.). Geomancy and Other Forms of Divination. Firenze: Sismel - Il Galluzzo, 2017. pp. 5-29.

CHARMASSON, T. Recherches sur une technique divinatoire: la géomancie dans l'Occident médiéval. Paris; Genève: Librarie Droz; Librarie Champion, 1980.

La condamnation parisienne de 1277. Texte latin, traduction, introduction et commentaire par D. Piché. Paris: Vrin, 1999.

CONTINI, G. Un poemetto provenzale di argomento geomantico. Fribourg: Librairie de l'Université, 1940.

DELATTE, A.; DELATTE, L. Un traité byzantin de géomancie. Codex Parisinus 2410. In: Mélanges Franz Cumont. Bruxelles: Université Libre de Bruxelles, 1936. pp. 575-658.

DOMINICUS GUNDISSALINUS. De divisione philosophiae. Ed. L. Baur. Münster i. W.: Aschendorff, 1903.

DOMINICUS GUNDISSALINUS. De divisione philosophiae. Über die Einteilung der Philosophie, Lateinisch - Deutsch, herausgegeben, übersetzt, eingeleitet und mit Anmerkungen versehen von A. Fidora, D. Werner. Freiburg i.B.: Herder, 2007.

Estimaverunt Indi. Firenze: Biblioteca Medicea Laurenziana, Plut. XXX.29, ff. 1-25.

FAHD, F. La divination arabe. Etudes religieuses, sociologiques et folkloriques sur le milieu natif de l'Islam. Leiden: Brill, 1966.

FAHD, T. khațt. In: Van DONZEL, E.; LEWIS, B.; PELLAT, Ch. (éd.). Encyclopédie de I'Islam. Nouvelle édition. Leiden: Brill, 1978. T. IV, coll.1160-1162.

FIDORA, A. Der wissenschaftliche Ort der Mantik in der Schule von Toledo (12. Jahrhundert). In: STURLESE, L. (hrsg. v.). Mantik, Schicksal und Freiheit im Mittelalter. Köln-Weimar-Wien: Böhlau, 2011. pp. 33-49.

FIDORA, A. Mantische Disziplinen als aristotelische Wissenschaft. Der epistemologische Integrationsversuch des Dominicus Gundissalinus. In: FIDORA, A. (hrsg. v.). Die mantischen Künste und die Epistemologie prognostischer Wissenschaften im Mittelalter. KölnWeimar-Wien: Böhlau, 2013. pp. 61-72.

FÜRBETH, F. Die Stellung der artes magicae in den hochmittelalterlichen 'Divisiones philosophiae'. In: SCHAEFER, U. (hrsg. v.). Artes im Mittelalter. Berlin: Akademie Verlag, 1999. pp. 249-262. 
FÜRBETH, F. Sandrichter und Dämonen in der Geomantie des Mittelalters. In: BOCKMANN, J.; GOLD, J. (hrsg. v.). Turpiloquium. Kommunikation mit Teufeln und Dämonen in Mittelalter und Früher Neuzeit. Würzburg: Königshausen \& Neumann, 2017. pp. 161-185.

GARIN, E. Lo zodiaco della vita. La polemica sull'astrologia dal Trecento al Cinquecento. Bari: Laterza, 1976.

GÁZQUEZ, J. M. The Attitude of the Medieval Latin Translators Towards the Arabic Sciences. Firenze: Sismel - Il Galluzzo, 2016.

GUILELMUS DE MORBECCA. Geomantia. In: RUBINO, E. Per una edizione della Geomantia di Guglielmo di Moerbeke: il testo del proemio e della prima distinzione della prima parte. In: PALAZZO, A.; ZAVATTERO, I. (ed.). Geomancy and Other Forms of Divination. Firenze: Sismel - Il Galluzzo, 2017. pp. 101-133.

HASKINS, C. H. Studies in the History of Mediaeval Science. Cambridge: Harvard University Press, 1924.

HEILES, M. Das Losbuch. Manuskriptologie einer Textsorte des 14. bis 16. Jahrhunderts. Wien-Köln-Weimar: Böhlau, 2018.

HUGO SANCTELLENSIS. Prologus super artem geomantiae. In: ARFÉ, P. Le dimore delle stelle nel Prologus super artem geomantiae di Ugo di Santalla. In: STRAFACE, A.; DE ANGELO, C.; MANZO A. (a cura di). Labor limae. Atti in onore di Carmela Baffioni, Studi Magrebini, n.s. 12/13, 2014/2015. pp. 41-72.

HUGO SANCTELLENSIS. Epilogus Artis geomantiae. In: ARFÉ, P. L'Ars geomantiae di Ugo di Santalla: il testo e la sua tradizione. In: PALAZZO, A.; ZAVATTERO, I. (ed.). Geomancy and Other Forms of Divination. Firenze: Sismel - Il Galluzzo, 2017. pp. 89-90.

IACOMO DELLA LANA, Commento alla "Commedia». A cura di M. Volpi con la collaborazione di A. Terzi. 4 tomi. Roma: Salerno Editrice, 2009.

IBN KHALDÛN. Discours sur l'histoire universelle: Al-Muqaddima. Traduction nouvelle, préface et notes par V. Monteil. 3 voll. Paris: Sindbad, 1978.

ISIDORUS HISPALENSIS. Etymologiarum sive originum libri XX. Ed. W. M. Lindsay, Oxford: Clarendon, 1911.

JAULIN, R. Essai d'analyse formelle d'un procédé géomantique. Bulletin de l'Institut Français d'Afrique Noire (series B), 19, pp. 43-71, 1957.

JAULIN, R. La géomancie: analyse formelle. Notes mathématiques de F. Dejean, R. Ferry. Paris; La Haye: Mouton \& CO, 1966.

KUNITZSCH, P. al-Manāzil. In: BOSWORTH, C.E.; VAN DONZEL, E.; LEWIS, B.; PELLAT, CH.; HEINRICHS, W.P. (ed.). The Encyclopaedia of Islam. New Edition. vol. VI, Leiden: Brill, 1991. pp. 374-376. 
MEANS, L. A translation of Martin of Spain's De geomancia. In: MATHESON, L. M. (ed.) Popular and Practical Science of Medieval England. East Lansing, Mich.: Colleagues Press, 1994. pp. 61-121.

MEYER, P. Traités en vers provençaux sur l'astrologie et la géomancie. Romania, v. 102, pp. 225-275, 1897.

PALAZZO, A. Geomancy as a Paradigm of Operative Rationality in the Middle Ages. Archiv für Mittelalterliche Philosophie und Kultur, v. 22, pp. 55-74, 2015.

PALAZZO, A. L'Estimaverunt Indi et la condanna del 1277. In: PALAZZO, A.; ZAVATTERO, I. (ed.). Geomancy and Other Forms of Divination. Firenze: Sismel - Il Galluzzo, 2017. pp. 167-221.

PALAZZO, A.; ZAVATTERO, I. (ed.). Geomancy and Other Forms of Divination. Firenze: Sismel - Il Galluzzo, 2017.

PEDRAZZI, M. Le figure della geomanzia: un gruppo finito abeliano. Physis. Rivista internazionale di storia della scienza, 14, n. 2, pp. 146-161, 1972.

PORRO, P. Divinazione e geomanzia in Tommaso d'Aquino: qualche osservazione sul De sortibus. In: PALAZZO, A.; ZAVATTERO, I. (ed.). Geomancy and Other Forms of Divination. Firenze: Sismel - Il Galluzzo, 2017. pp. 143-166.

RODRÍGUEZ-ARRIBAS, J. Divination According to Goralot: Lots and Geomancy in Hebrew Manuscripts. In: PALAZZO, A.; ZAVATTERO, I. (ed.). Geomancy and Other Forms of Divination. Firenze: Sismel - Il Galluzzo, 2017. pp. 243-270.

RUBINO, E. An Italian Translation of William of Moerbeke's Geomancy. Medioevo. Rivista di Storia della Filosofia Medievale, v. 42, pp. 81-98, 2017.

SAVAGE-SMITH, E.; SMITH, M. B. Islamic Geomancy and a Thirteenth-Century Divinatory Device. Malibu: Undena Publications, 1980.

SIGERUS DE BRABANTIA. Quaestiones in Metaphysicam (reportatio di Cambridge). Ed. A. Maurer. Louvain-la-Neuve: Éditions de l'Institut supérieur de philosophie, 1983. (Philosophes Médiévaux, 25).

SKINNER, S. Terrestrial Astrology. Divination by Geomancy. London; Boston: Routledge \& Keagan Paul, 1980.

SMITH, M. B. The Nature of Islamic Geomancy with a Critique of a Structuralist's Approach. Studia Islamica, v. 49, pp. 5-38, 1979.

STURLESE, L. Thomas von Aquin und die Mantik. In: STURLESE, L. (hrsg. v.). Mantik, Schicksal und Freiheit im Mittelalter. Köln-Weimar-Wien: Böhlau, 2011. pp. 97-107.

TANNERY, P. L'introduction de la géomancie en Occident et le traducteur Hugo Sanccelliensis, II. In: TANNERY, P. Le Rabolion, in Mémoires Scientifiques, vol. IV. Toulouse; Paris: Édouard Privat; Gauthier-Villars, 1920. pp. 318-53. 
THOMAS DE AQUINO. Liber de sortibus ad dominum Iacobum de Tonengo. Cura et studio Fratrum Praedicatorum. Roma: 1976. (Sancti Thomae de Aquino Opera Omnia, 43, pp. 203-241).

THOMAS DE AQUINO. Summa theologiae. Cinisello Basalmo. Milano: Edizioni San Paolo, 1988.

VAN BINSBERGEN, W. The Astrological Origin of Islamic Geomancy, paper read at: The SSIPS (Society for the Study of Islamic Philosophy and Science). SAGP (Society of Ancient Greek Philosophy). In: GLOBAL AND MULTICULTURAL DIMENSIONS OF ANCIENT AND MEDIEVAL PHILOSOPHY AND SOCIAL THOUGHT, $15^{\text {th }}$. Annual Conference New York, 1997, pp. 1-63. Disponível em: http://studylib.net/ doc/1885 3949/the-astrological-origin-of-islamic-geomancy. Acesso em: 15 Au. 2019 (unpublished).

VILLUENDAS SABATÉ, B. Arabic geomancy in Jewish hands. Specimens from the Cairo Genizah. In: PALAZZO, A.; ZAVATTERO, I. (ed.). Geomancy and Other Forms of Divination. Firenze: Sismel - Il Galluzzo, 2017. pp. 271-288.

ZAVATTERO, I. Estimaverunt Indi: la diffusion d'un text geomantique condamné. Medioevo: Rivista di Storia della Filosofia Medievale, v. 42, pp. 57-80, 2017.

ZAVATTERO, I. Estimaverunt Indi: la tradizione testuale di un anonimo trattato di geomanzia. In: PALAZZO, A.; ZAVATTERO, I. (ed.). Geomancy and Other Forms of Divination. Firenze: Sismel - Il Galluzzo, 2017. pp. 31-63.

ZIEGLER, A. K. Histoire de la géomancie latine du milieu du XII ${ }^{e}$ siècle au milieu du $\mathrm{XVI}^{\mathrm{e}}$ siècle. In: Positions des thèses soutenues par les élèves de la promotion de 1934 pour obtenir la diplôme d'archiviste paléographe. Paris: École Nationale des Chartes, 1934. pp. 159-170.

Recebido: 30/12/2019

Aceito: 30/12/2019 
PALAZZO, A 